\title{
Role of Diffusion Weighted Magnetic Resonance Imaging in Evaluation of Patients with Salivary Glands Tumors
}

\author{
Abdallah Mohammed AL-kheshen, Mohammed Salah EL-Feshawy, Mohammed Essmat Abd- \\ ElGhani, Mahmoud Mohammed Abomosalam Ali
}

\author{
Department of Radiodiagnosis, Faculty of Medicine, Al-Azhar University \\ Corresponding author: Mahmoud Mohammed Abomosalam Ali, Mobile: 002001140268464, EMail: \\ mabomosalam87@gmail.com
}

\begin{abstract}
:
Background: The diffusion weighted imaging and ADC value give appreciable information about tumor cellularity with tissue contrast between the active and necrotic areas within the tumor. This would be valuable and could direct the beneficial site for interventional procedure and biopsy to be taken from the viable tumoral tissue especially in case with non-enhanced studies due to renal impairment.

Objective: The aim of this study was to investigate the capacity of Diffusion MRI to predict the benign and malignant Salivary gland tumors, using ADC value and DWI.

Patients and Methods: This study included 40 patients (24 male and 16 female). Sixteen patients had malignant lesions and twenty-four had benign ones. The DWI was obtained with $b$ values including 0 and $800 \mathrm{~mm}^{2} /$ second. The Apparent Diffusion Coefficient (ADC) generated by measuring identical images at different b-values and represented as ADC map, from which the ADC value was calculated.

Results: The absolute ADC value of lesions was significantly different between benign and malignant salivary gland tumors $(\mathrm{p}<0.001)$. The sensitivity of ADC in differentiating benign from malignant lesions in our study was $93.7 \%$ indicating a high true positive rate. Hence, if the average ADC is below $0.85 \times 10^{-3} \mathrm{~mm}^{2} / \mathrm{sec}$, there is high probability that the mass will be malignant with high specificity of $95.8 \%$. Results revealed that the mean ADC value of benign and malignant salivary gland tumors were $1.33 \pm 0.46 \times 10^{-3} \mathrm{~mm}^{2} / \mathrm{s}$ and $0.65 \pm 0.21 \times 10^{-3} \mathrm{~mm}^{2} / \mathrm{s}$, respectively. The mean ADC value of benign was significantly higher than that of malignant lesions.
\end{abstract}

Conclusion: The use of DWI and ADC values can provide better assessment of salivary gland tumors and predict the benign and malignant lesions.

Keywords: ADC, MRI, DWI, Malignant Salivary Gland Tumors

\section{INTRODUCTION}

The major salivary glands are the parotid glands, submandibular glands and sublingual glands. There are also a large number (600$1,000)$ of minor salivary glands widely distributed throughout the oral mucosa, palate, uvula, floor of the mouth, posterior tongue, retro molar and per tonsillar area, pharynx, larynx and paranasal sinuses. Tumors affecting salivary glands may be benign or malignant and are diverse in their pathology. About $80 \%$ of salivary gland tumors occur in the parotid gland (1).

The majority of salivary neoplasms are benign (65-70\%). Nearly 8o\% of parotid gland tumours is benign. Malignant tumours are rare, Malignancy typically presents after age 6o, whilst benign lesions usually occur after age 40 (2). Only $5 \%$ of all salivary gland tumours occur in childhood ${ }^{(3)}$.
Surgical approach to parotid tumors is different for benign and malignant neoplasms, but the clinical symptoms do not correlate well with histology and only few symptoms, as facial palsy, allow the diagnosis of malignancy. Therefore, preoperative imaging has assumed a major role in surgical planning for assessing the location and malignancy of the tumor ${ }^{(4)}$.

Pre-operative diagnosis of the type of salivary gland mass can help the surgeon determine the most suitable surgical procedure. MR imaging (MRI) is the method of choice for the characterization of salivary gland tumors. MRI is a non-invasive technique that can provide morphological information of the mass, allow correct diagnosis and proper staging. It can accurately determine the deep or superficial location of the mass, extension, contour and signal pattern ${ }^{(5)}$. 
New MRI techniques including dynamic contrast-enhanced MRI (DCE-MRI), diffusionweighted MRI (DW-MRI) have shown promising results in the differentiation between benign and malignant gland tumors in addition to the static MR imaging ${ }^{(5)}$.

Diffusion-weighted imaging (DWI) provides functional information related to the random water diffusion of the mass and has a high ability to determine different histologic subtypes of salivary gland tumors. Apparent diffusion coefficient (ADC) values calculated from DWI can provide quantitative information related to random diffusion of water molecules in tissues and functionally complement conventional MRI and has been reported as helpful for narrowing the differential diagnosis of salivary gland masses ${ }^{(6)}$.

The aim of this study was to investigate the capacity of Diffusion MRI to predict the benign and malignant Salivary gland tumors, using ADC value and DWI.

\section{PATIENTS AND METHODS}

This cross sectional study included a total of 40 patients (24 male and 16 female), sixteen had malignant lesions and twenty-four had benign ones, attending at Radio diagnosis Department, El-Hussein University Hospital and National Cancer Institute. Approval of the ethical committee and a written informed consent from all the subjects were obtained. This study was conducted between March 2017 to September 2018.

\section{Patient inclusion criteria:}

1- any age group and sex

2- Patient with clinical and radiological findings suggestive of salivary gland tumours.

3- The lesion has solid portions suitable for region of interest (ROI) analysis.

\section{Patient exclusion criteria:}

1- Totally cystic lesions.

2- Patients with metallic implants.

3- Patients with claustrophobia.

4- Patients on electrically programmed infusion pump.

All patients were subjected to: Complete history taking, full clinical examination, ultrasound examination and MRI examination.

Machines used: MRI Cheiva Phillips 1.5 Tesla.

\section{Parameters of diffusion:}

Axial DW imaging was performed by using a single-shot T2-weighted echo planar spin-echo sequence with the following parameters: 1600/107; diffusion gradient encoding in three $(\mathrm{x}, \mathrm{y}, \mathrm{z})$ orthogonal directions; $b$ values of 0 and 800 $\mathrm{sec} / \mathrm{mm}^{2}$; field of view, $220 \mathrm{~mm}$; matrix size, 128 - 128; section thickness, 4mm; section gap, $0 \mathrm{~mm}$; and one signal acquired. At each $b$ value, $\mathrm{x}, \mathrm{y}$, and $\mathrm{z}$ single-direction DW images and a baseline image $\left(b_{-} 0 \mathrm{sec} /\right.$ $\left.\mathrm{mm}^{2}\right)$ were acquired; combined $([\mathrm{x}-\mathrm{y}$ z]/3) DW imaging was calculated and performed automatically by the MR instrument.

All DW imaging data were transferred to a computer workstation for determination of the signal intensity and ADC. Each image used for the creation of the ADC maps was obtained with one signal acquired.

The ADC values were measured by manually placing regions of interest "ROI" in tumor regions on the ADC map. Whenever possible, regions of interest were placed in at the site of enhanced lesions on contrast-enhanced T1- weighted MR images, also taking in consideration other MR images to carefully place the regions of interest only in the solid tumor components. Thus, cystic, necrotic, and hemorrhagic tumor areas were excluded.

\section{Statistical Analysis:}

Recorded data were analyzed using the statistical package for social sciences, version 20.0 (SPSS Inc., Chicago, Illinois, USA). Quantitative data were expressed as mean \pm standard deviation (SD). Qualitative data were expressed as frequency and percentage.

The following tests were done:

- Independent-samples t-test of significance was used when comparing between two means.

- Chi-square $\left(\mathrm{x}^{2}\right)$ test of significance was used in order to compare proportions between two qualitative parameters.

- Receiver operating characteristic (ROC curve) analysis was used to find out the overall productivity of parameter in and to find out the best cut-off value with detection of sensitivity and specificity at this cut-off value. 
- The confidence interval was set to $95 \%$ and the margin of error accepted was set to $5 \%$. So, the p-value was considered significant as the following:

- Probability (P-value)
- P-value $<0.05$ was considered significant.

- P-value $<0.001$ was considered as highly significant.

- P-value >0.05 was considered insignificant.

\section{RESULTS:}

Demographic data:

This study included 40 cases (24 Male and 16 Female) patients.

Patient ages ranged from 10-80 years with mean of (45 years).

Table (1): Demographic data distribution of the study group ( $\mathrm{N}=40)$.

\begin{tabular}{|c|c|c|}
\hline Demographic Data & No. & $\%$ \\
\hline \multicolumn{3}{|l|}{ Age (years) } \\
\hline$<35$ years & 12 & 30 \\
\hline $35-60$ years & 20 & 50 \\
\hline$>60$ years & 8 & 20 \\
\hline Range $[$ Mean \pm SD] & \multicolumn{2}{|c|}{$30-70[48.40 \pm 14.28]$} \\
\hline Sex & & \\
\hline Female & 16 & 40 \\
\hline Male & 24 & 60 \\
\hline
\end{tabular}

This table shows that the $<35$ years (30\%), 35-60 years (50\%) and >60 years (20\%), of age, also female $(40 \%)$ and male $(60 \%)$ of sex.

Location of the lesions:

$80 \%$ of our cases were found in the parotid gland, $10 \%$ in the submandibular, $10 \%$ in the minor salivary gland (hard palat).

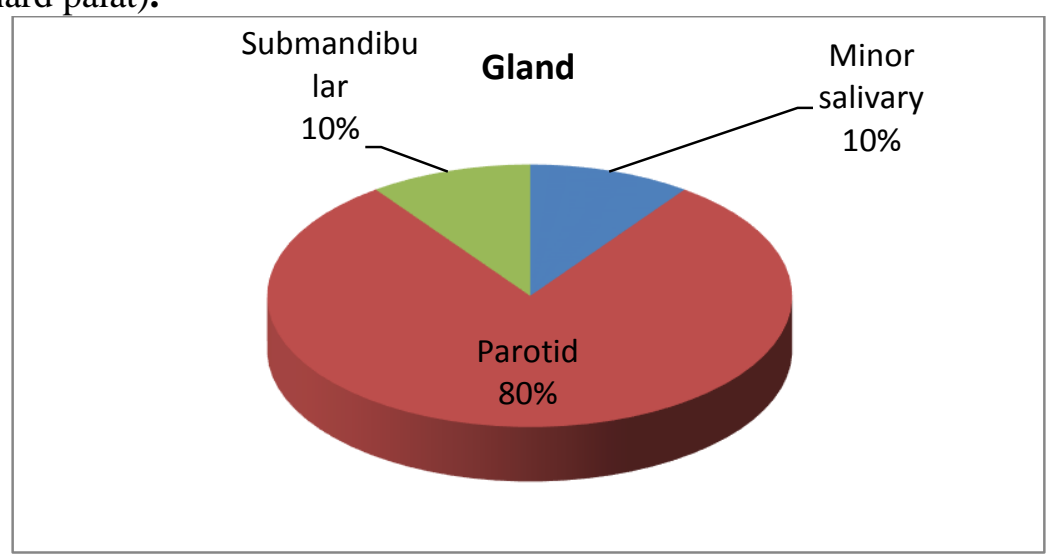

Fig. (1): Pie chart gland distribution of the study group $(\mathrm{N}=40)$.

\section{Pathological diagnosis:}

Patients were distributed according to final pathological diagnosis into 2 groups; the benign group and the malignant group. The benign group included 24 patients while the malignant group included 16 patients.

The diagnoses in the benign group as determined by pathological analysis were: pleomorphic adenoma, Warthin tumor, lipoma, basal cell adenoma.

Pleomorphic adenoma showed to be the most frequent among benign lesions representing $50 \%$ of all benign lesions.

Whereas malignant group shows the following entities: lymphoma, adenoid cystic carcinoma, basal cell carcinoma.

However, the most common malignant lesions found in our study were lymphoma "representing $50 \%$ of all malignant tumors.

Table (2): Histopathology distribution of the study group $(\mathrm{N}=40)$. 


\begin{tabular}{|l|c|c|}
\hline Histopathology & No. & \% \\
\hline Malignant & $\mathbf{1 6}$ & $\mathbf{4 0}$ \\
Adenoid cystic carcinoma & 4 & 10 \\
Basal cell carcinoma & 4 & 10 \\
Lymphoma & 8 & 20 \\
\hline Benign & $\mathbf{2 4}$ & $\mathbf{6 0}$ \\
Basal cell adenoma & 2 & 8 \\
Lipoma & 4 & 17 \\
Pleomorphic adenoma & 16 & 67 \\
Whartons Tumor & 2 & 8 \\
\hline
\end{tabular}

This table shows that the Malignant (40\%), Adenoid cyctic carcinoma (10\%), Basal cell carcinoma (10\%), Lymphoma (20\%), Benign (60\%), Basal cell adenoma (8\%), Lipoma (17\%), Pleomorphic adenoma (67\%) and Whartons Tumor (8\%) of histopathology.

\section{Conventional finding:}

All benign tumors seen low T1, High T2 and High STIR Except, Lipoma.

lipoma seen high T1 and T2 and Low in STIR .

Malignant tumors seen low or intermediate T1 and High T2 except lymphoma seen low T2 .

All of malignant tumors were enhanced.

Table (3): Spread distribution of the study group ( $\mathrm{N}=40)$.

\begin{tabular}{|l|c|c|}
\hline Spread & No. & \% \\
\hline No Spread & 24 & 60 \\
\hline Perineural extention through facial nerve & 8 & 20 \\
\hline Enlarged cervical lymph nodes & 8 & 20 \\
\hline Total & 40 & 100 \\
\hline
\end{tabular}

This table shows that No Spread (60\%), Perineural extension through facial N. (20\%), enlarged cervical lymph nodes (20\%) of spread.

\section{Diffusion finding:}

Benign tumors seen facilitated in diffusion and T2 shine through except (Wharton's tumor and basal cell adenoma) seen restricted.

ADC value of benign tumors were High (0.7-1.8), highest ADC value was (pleomorphic adenoma 1.8), lowest ADC value (basal cell adenoma 0.7).

Malignant tumors were restricted in the diffusion.

ADC value of malignant tumors were low $(0.49-1)$, lowest ADC was (lymphoma 0.4).

Table (4): Relation between pathological diagnosis and diffusion findings.

\begin{tabular}{|c|c|c|c|c|c|c|}
\hline \multirow{3}{*}{ Diffusion findings } & \multicolumn{4}{|c|}{ Pathological diagnosis } & \multicolumn{2}{|c|}{ Chi-square test } \\
\hline & \multicolumn{2}{|c|}{ Malignant $(\mathrm{N}=16)$} & \multicolumn{2}{|c|}{ Benign $(\mathrm{N}=24)$} & \multirow{2}{*}{$\mathbf{x} 2$} & \multirow{2}{*}{ p-value } \\
\hline & No. & $\%$ & No. & $\%$ & & \\
\hline \multicolumn{7}{|l|}{ DWI } \\
\hline $\begin{array}{l}\text { High } \\
\text { Low }\end{array}$ & $\begin{array}{c}16 \\
0\end{array}$ & $\begin{array}{c}100.0 \% \\
0.0 \%\end{array}$ & $\begin{array}{c}16 \\
8\end{array}$ & $\begin{array}{l}66.7 \% \\
33.3 \%\end{array}$ & 6.667 & $0.010^{*}$ \\
\hline \multicolumn{7}{|l|}{ ADC map } \\
\hline $\begin{array}{l}\text { High } \\
\text { Low }\end{array}$ & $\begin{array}{c}0 \\
16\end{array}$ & $\begin{array}{c}0.0 \% \\
100.0 \%\end{array}$ & $\begin{array}{c}16 \\
8\end{array}$ & $\begin{array}{l}66.7 \% \\
33.3 \% \\
\end{array}$ & 17.778 & $<0.001 * *$ \\
\hline \multicolumn{7}{|l|}{$\begin{array}{l}\text { ADC value (x10-3 } \\
\text { mm2/sec) }\end{array}$} \\
\hline $\begin{array}{l}\text { Range } \\
{[\text { Mean } \pm \text { SD }]}\end{array}$ & \multicolumn{2}{|c|}{$\begin{array}{c}0.49-1.00 \\
{[0.65 \pm 0.21]}\end{array}$} & \multicolumn{2}{|c|}{$\begin{array}{c}0.70-1.80 \\
{[1.33 \pm 0.46]}\end{array}$} & 6.225 & $<0.001 * *$ \\
\hline
\end{tabular}

$x^{2}:$ Chi-square test;

$* p$-value $<0.05 \mathrm{~S} ; * * p$-value $<0.001 \mathrm{HS}$

This table shows statistically significant overlap between malignant and benign according to DWI, ADC map and ADC value.

Accuracy of DWI

Lesions were assessed with DWI and ADC map. They were labelled as either benign-looking "B" or malignant-looking " $\mathrm{M}$ ", according to presence of criteria of malignancy; e.g. high signal in 
DWIs "i.e. restricted diffusion pattern" and low ADC value. By DWI and ADC map 20 malignantlooking lesions were detected, after pathological analysis; only 16 proved to be truly positive for malignancy with 4 false positive cases. 20 lesions were detected as benign looking; 20 proved to be truly negative for malignancy (benign), with no false negative ones. Thus, using DWI in predicting benign and malignant lesions has the sensitivity of $93.7 \%$ and specificity of $95.8 \%$ with positive predictive value $93.8 \%$ negative predicative value $95.8 \%$ with diagnostic accuracy $94.4 \%$.

Table (5): Diagnostic Performance of ADC value $\left(\times 10^{-3} \mathrm{~mm} 2 / \mathrm{sec}\right)$ in Discrimination of malignant and benign.

\begin{tabular}{|c|c|c|c|c|c|}
\hline Cut-off & Sen. & Spe. & PPV & NPV & Accuracy \\
\hline$<\mathbf{0 . 8 5}$ & 93.7 & 95.8 & 93.8 & 95.8 & $94.4 \%$ \\
\hline
\end{tabular}

Receiver operating characteristics (ROC) curve was used to define the best cut off value of ADC value was $<0.85$, with sensitivity of $93.7 \%$ specificity of $95.8 \%$ positive predictive value of $93.8 \%$, negative predictive value of $95.8 \%$ with diagnostic accuracy of $94.4 \%$.

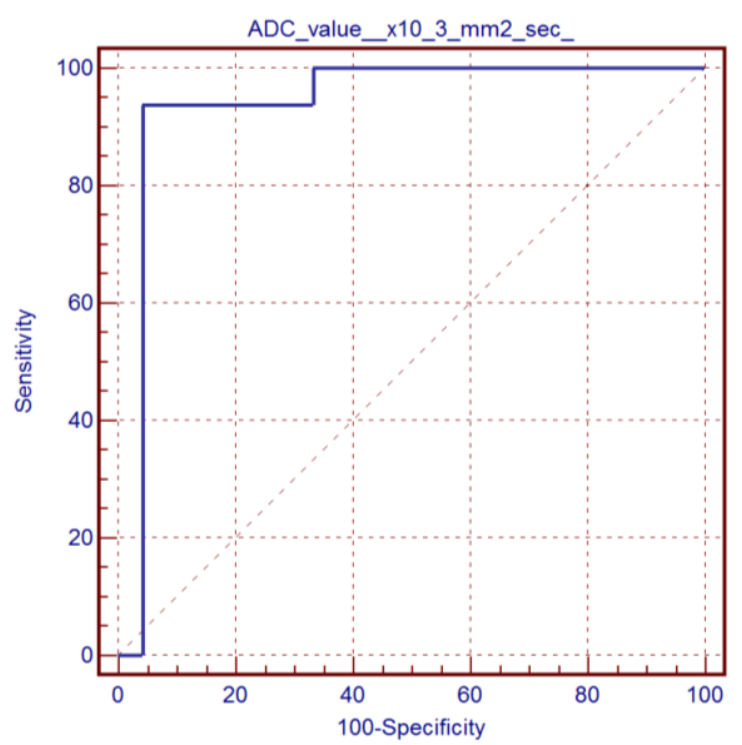

Fig. (2): ROC curve for prediction of malignant tumors.

\section{DISCUSSION}

This study we included 4o patients (24 male and 16 female). Sixteen patients had malignant lesions and twenty four had benign ones. The DWI was obtained with $b$ values including $o$ and $800 \mathrm{~mm}^{2} / \mathrm{second}$. The Apparent

In our study, the absolute ADC value of lesions was significantly different between benign and malignant salivary gland tumors $(\mathrm{p}<0.001)$.

The sensitivity of ADC in differentiating benign from malignant lesions in our study was 93.7\% indicating a high true positive rate. Hence, if the average ADC is below $0.85 \times 10^{-3}$ $\mathrm{mm}^{2} / \mathrm{sec}$, there is high probability that the mass will be malignant with high specificity of 95.8 $\%$.

Our results revealed that the mean ADC value of benign and malignant salivary gland tumors were $1.33 \pm 0.46 \times \mathrm{x}^{-3} \mathrm{~mm}^{2} / \mathrm{s}$ and
Diffusion Coefficient (ADC) generated by measuring identical images at different bvalues and represented as ADC map, from which the ADC value was calculated.

$0.65 \pm 0.21 \times 10^{-3} \mathrm{~mm}^{2} / \mathrm{s}$, respectively. The mean $\mathrm{ADC}$ value of benign was significantly higher than that of malignant lesions.

In agreement of the results of our study is Salama et al. ${ }^{(7)}$ a study that was performed on 25 patients with parotid gland masses (11 males of cases and 14 females; age ranged from 22 to 79 years; mean age was $53.4 \pm 13.6$ years).

The benign masses were $18(72 \%)$. The most common benign tumors were pleomorphic adenomas (61.12\% of benign tumors), followed by Warthin tumor (38.88\% of benign tumors). The malignant tumors were 7 (28\%). The most common malignant tumors were muco 
epidermoid carcinomas representing $71.43 \%$ off all malignant tumors and, being more frequently seen in males ${ }^{(7)}$.

They concluded that ADC of malignant lesions were significantly lower than that of benign lesions, with the exception of Warthin's tumour. As the mean ADC of malignant tumors $(1.03 \pm 0.13 \times 103 \mathrm{~mm} 2 / \mathrm{s})$ has been shown to be significantly smaller than that of pleomorphic adenomas $(1.89 \pm 0.18 \cdot 103 \mathrm{~mm} 2 / \mathrm{s})$, however there was an overlap between the mean ADC values of Warthin tumors $\left(0.92 \pm 0.22 \times 10^{-3}\right.$ $\left.\mathrm{mm}^{2} / \mathrm{s}\right)$ and that of malignant tumors $(1.03 \pm$ $\left.0.13 \times 10^{-3} \mathrm{~mm}^{2} / \mathrm{s}\right)^{(7)}$.

The mean ADC value for malignant tumors was $1.03 \pm 0.13 \times 10^{-3} \mathrm{~mm} 2 / \mathrm{s}$. The malignant masses showed low ADC values (0.6 $\pm 1.2 \times 10^{-3} \mathrm{~mm}^{2} / \mathrm{s}$ ) ${ }^{(7)}$.

In our study the mean ADC value for malignant lesions was $0.65 \pm 0.21 \times 10^{-3} \mathrm{~mm}^{2} / \mathrm{s}$, with range of $\left(0.49-0.21 \times 10^{-3} \mathrm{~mm}^{2} / \mathrm{s}\right)$.

The minute difference in range and mean values in our study; may be attributed to the fact that the number of lymphoma cases were more in our study, thus lowering the mean ADC value.

This is familiar in literature, since lymphoma records particularly low ADC values, with a ratio often inferior to $0.5^{(8)}$.

They concluded that: ADC value may provide preoperative tissue characterization of the salivary gland tumors.

They also observed that in contrast to salivary gland cancers, malignant lymphomas arising in the salivary glands were associated with extremely low ADCs (ADC value is lower than $0.6 \times 10^{-3} \mathrm{~mm}^{2} / \mathrm{s}$ ) throughout the lesions this was consistent with the homogeneous growth patterns of lymphoma cells ${ }^{(9)}$.

This agrees with our observations in our study as the mean $\mathrm{ADC}$ value for lymphoma was $0.55 \pm 0.16 \times 10^{-3} \mathrm{~mm}^{2} / \mathrm{s}$.

In Balçık et al. ${ }^{(10)}$, they included a total of 41 parotid gland masses in the 4o patients (22 females, 18 males; age ranging between 16-85 years; mean age, $51.5 \pm 19.1$ years) in this study. Six of these had been diagnosed by histopathological examination. One mass (lipoma) had been diagnosed via pathognomonic MRI and CT features.

Pathological diagnosis of these lesions were also diverse including pleomorphic adenoma , Warthin's tumor, basal cell adenoma, dermoid cyst, cyst that was full of keratinized material, lipoma, mucoepidermoid carcinoma, adenoid cystic carcinoma, carcinoma of the salivary duct, adenocarcinoma, carcinoma ex-pleomorphic adenoma , squamous cell carcinoma and secondary tumors.

While, the most common benign parotid gland lesion was pleomorphic adenoma, secondary tumors were the most determined lesions within the malignant tumors.

The mean ADC value of benign lesions was measured as $1.74 \pm 0.58 \times 10^{-3} \mathrm{~mm}^{2} / \mathrm{s}$, malignant ones was $1.13 \pm 0.13 \times 10^{-3} \mathrm{~mm}^{2} / \mathrm{s}$ and the mean ADC values of benign lesions were significantly higher than malignant lesions $(p=0.006)$. This agrees with our study results.

Malignant lesions were fewer in number thus were not compared among themselves.

In the comparisons that were performed between pleomorphic adenoma (group 1), Warthin's tumor (group 2) and malignant tumors (group 3). The ADC values of the pleomorphic adenoma were significantly higher than malignant tumors $(p<0.001)$ or Warthin's tumors $(p=0.001)$. The ADC values of malignant tumors were significantly higher than Warthin's tumors $(\mathrm{p}=0.001)$.

Balçık et al. (10) concluded no absolute optimal cut off, preferring to separate the cut off between dual groups of pleomorphic adenoma, warthins, and malignant lesion. Thus they proposed two numbers:

Accordingly, the pleomorphic adenomas were differentiated from all other benign and malignant tumors with $1.60 \times 10^{-3} \mathrm{~mm}^{2} / \mathrm{sec}$ cutoff ADC value that had sensitivity of $94.7 \%$ $(18 / 19,95 \%$ CI $)$ and specificity of $100 \%$ $(22 / 22,95 \% \mathrm{CI})$.And The malignant tumors were differentiated from Warthin's tumours with $1.01 \times 10^{-3} \mathrm{~mm}^{2} / \mathrm{sec}$ cutoff ADC value that had sensitivity of $92.3 \%(12 / 13,95 \%$ CI $)$ and specificity of $100 \%(4 / 4,95 \% \mathrm{CI})$.

This agrees with the recorded low ADC value for Warthin's tumor in our study which was $0.8 \times 10-3 \mathrm{~mm}^{2} / \mathrm{sec}$ which is considered a false positive result on the basis of ADC value. However, owing to paucity of cases in this category, no dual comparison between Warthin's tumor group and malignant lesions group was done.

This finding might be attributed to the intense lymphoid accumulation in the stroma and proliferation of the epithelial component leading to a decrease in the extracellular extravascular space and therefore a decrease in $\mathrm{ADC}$, even less than malignant lesions ${ }^{(7)}$. 
The major limitation of our study was that it has been done on several salivary glands which offer naturally diverse histological profiles, thus the numbers of the lesions in each category were few. So further separate studies dedicated to different pathological entities may provide differentiation between tumor types.

In our study there was a striking false result on basis of DWI and ADC value, the patient had a parotid Warton's tumor and basal cell adenoma. The calculated ADC value was $0.8 \times 10^{-3} \mathrm{~mm}^{2}, 0.7 \times 10^{-3} \mathrm{~mm}^{2} / \mathrm{s}$ respectively, which may be mistaken as malignant lesion.

This can be explained by the fact that this lesion is composed of a diffused, wellorganized lymphoid tissue and lymphocytic interstitial infiltrate ${ }^{\mathbf{( 1 1 )}}$.

\section{CONCLUSION}

In conclusion, the use of DWI and ADC values can provide better assessment of salivary gland tumors and predict the benign and malignant lesions.

Thus, we recommend using DWI as part of the routine exam of salivary gland tumors as well as integrating the ADC value as part of the regular interpretation by radiologists. Since DWI is a non-invasive method and has the advantage of not being time consuming, nor does it require additional special equipment. Yet it represents valuable addition to the conventional MRI examination adding up considerably to its efficacy as discussed before.

\section{REFERENCES}

1- Namboodiripad PC (2014): A review: Immunological markers for malignant salivary gland tumors. J. Oral Biol. Craniofac. Res., 4(2):127-34.

2- Mehanna H, McQueen A and Robinson M. (2012): Salivary gland swellings. BMJ., 345:6794.

3- Iro H. and Zenk J. (2014): Salivary gland diseases in children. GMS current topics in otorhinolaryngology. Head and Neck Surgery, 13:

4- Christe A, Waldherr $\mathbf{C}$ and Hallett $\mathbf{R}$ (2011): MR imaging of parotid tumors: Typical lesion characteristics in MR imaging improve discrimination between benign and malignant disease. A.J.N.R., 32:1202 - 1207.

5- Yerli H, Aydin E, Haberal N et al. (2010): Diagnosing common parotid tumors with magnetic resonance imaging including diffusion-weighted imaging vs. fine-needle aspiration cytology: A comparative study. Dentomaxillofac Radiol., 39(6): 349 - 355.

6- Buijs M, Vossen JA, Hong K, Kamel IR et al. (2012): Chemoembolization of hepatic metastases from ocular melanoma assessment of response with contrastenhanced and diffusion weighted MRI. A.J.R. Am. J. Roentgenol., 191(1):285-9.

7- Salama AA, El-Barbary AH, Mlees MA et al. (2014): Value of apparent diffusion coefficient and magnetic resonance spectroscopy in the identification of various pathological subtypes of parotid gland tumors. The Egyptian Journal of Radiology and Nuclear Medicine, 46(1): 45-52.

8- Thiagarajan S, Sudhir NV and Poonam J (2014): A review of salivary gland neoplasms and its management. Tata Memorial Centre Mumbai India, 4(3): 1636.

9- Eida S, Sumi M, Sakihama $\mathbf{N}$ et al. (2007): Apparent diffusion coefficient mapping of salivary gland tumors: prediction of the benignancy and malignancy. A.J.N.R. Am. J. Neuroradiol., 28: $116-121$.

10- Balçık C, Akan H and İncesu L. (2014): Evaluating of parotid gland tumours according to diffusion weighted MRI. Eur. J. Gen. Med., 11(2): 77-84.

11- Orme IM and Basaraba RJ (2014): The formation of the granuloma in tuberculosis infection. Semin. Immunol., 26(6):601-9. 